\title{
Friedberg numberings in the Ershov hierarchy
}

\author{
Serikzhan A. Badaev, Mustafa Manat ${ }^{\dagger}$ and Andrea Sorbi ${ }^{\ddagger}$
}

\begin{abstract}
We show that for every $n \geq 1$, there exists a $\Sigma_{n}^{-1}$-computable family which up to equivalence has exactly one Friedberg numbering which does not induce the least element of the corresponding Rogers semilattice.
\end{abstract}

\section{Introduction}

Minimal numberings became a fashionable research topic in the classical theory of numberings at the end of the sixties. The study of minimal numbering beginning from the famous theorem of Friedberg [10] on the existence of oneto-one computable numbering of the family $\mathcal{C}$ of all c.e. sets. One of the main questions on minimal numberings, that is the problem of finding, up to equivalence of numberings, the possible number of minimal numberings, was settled by Yu.L. Ershov[3]. A Friedberg numbering is a special but very important case of minimal numbering. The theory of minimal numberings, and in particular Friedberg numberings, has many successful applications in classical recursion theory, recursive model theory ([9], [14]), and theoretical computer science ([15]).The main powerful methods for constructing families of c.e. sets with a finite number of Friedberg numberings, due to Goncharov [9], to show that numbers of spectrum of the nonautoequivalent constructivizations of recursive models is equal to $\{\omega, 0,1,2 \ldots\}$ ([12]). It was the starting point of some of the most important researches on algorithmic dimension of recursive models. Another application of this results was found by Kummer ([15]).

We refer to Kleene's system $O$ of ordinal notations for computable ordinals: for details, see [18]. In particular, for $a \in O$, the symbol $|a|_{O}$ represents the

\footnotetext{
*Al-Farabi Kazakh National University, Al-Farabi ave., 71, Almaty, 050038, Kazakhstan. Part of the research contained in this paper was carried out while the first author was GNSAGA-INDAM Visiting Professor at the Department of Mathematics and Computer Science "Roberto Magari" of the University of Siena, Italy, July 2011. The first author wishes to thank INDAM-GNSAGA for supporting the visiting professorship.

${ }^{\dagger}$ Al-Farabi Kazakh National University, Al-Farabi ave., 71, Almaty, 050038, Kazakhstan. Part of the research contained in this paper was carried out while the second author was visiting the Department of Mathematics and Computer Science "Roberto Magari" of the University of Siena, Italy. The second author wishes to thank the Al-Farabi University for supporting the visit,and the Department of Mathematics and Computer Science "Roberto Magari" of siena for its hospitality.

${ }^{\ddagger}$ Dipartimento di Scienze Matematiche ed Informatiche "Roberto Magari", Università di Siena, 53100 Siena, Italy.
} 
ordinal of which $a$ is a notation; the symbol $<_{O}$ denotes Kleene's partial ordering relation on $O$; moreover, the symbol $+_{0}$ denotes a partial computable function, defined on $O$, such that $\left|a+_{O} b\right|_{O}=|a|_{O}+|b|_{O}$, and $a \leq_{O} a+_{O} b$.

Definition 1.1. Any surjective mapping $\alpha$ of the set $\omega$ of natural numbers onto a nonempty set $A$ is called a numbering of $A$. Let $\alpha$ and $\beta$ be numberings of $A$. We say that numbering $\alpha$ is reducible to numbering $\beta$ (in symbols, $\alpha \leqslant \beta$ ) if there exists a computable function $f$ such that $\alpha(n)=\beta f(n)$ for any $n \in \omega$. We say that the numberings $\alpha$ and $\beta$ are equivalent (in symbols, $\alpha \equiv \beta$ ) if $\alpha \leqslant \beta$ and $\beta \leqslant \alpha$.

Definition 1.2. Let $\theta_{\alpha} \rightleftharpoons\{\langle x, y\rangle \mid \alpha x=\alpha y\}$. Numbering $\alpha$ is called decidable (positive) if $\theta_{\alpha}$ is decidable (correspondingly, c.e.) set.Numbering $\alpha$ is called Fridberg if it is one to one.

It is obvious that if $\alpha$ and $\beta$ are equivalent numberings then $\alpha$ is decidable (positive) if and only if $\beta$ is decidable (positive). Every decidable numbering of infinite family is equivalent to one-to-one numbering or single-valued numbering $[7],[8]$.

Rogers semilattice $\mathcal{R}_{a}^{i}(\mathcal{A})$ of a family $\mathcal{A} \subseteq \Sigma_{a}^{i}$ is a quotient structure of all $\Sigma_{a}^{i}$-computable numberings of the family $\mathcal{A}$ modulo equivalence of the numberings ordered by the relation induced by reducibility of the numberings. $\mathcal{R}_{a}^{i}(\mathcal{A})$ allows one to measure the different computations of a given family $\mathcal{A}$ and used also as a tool to classify the properties of $\Sigma_{a}^{i}$-computable numberings for the different families $\mathcal{A}$.

A numbering $\alpha$ of a set $A$ is said to be minimal if $\beta \leq \alpha$ implies $\alpha \leq \beta$ for every numbering $\beta$ of $A$. The minimal numberings are just those ones which induce the minimal elements in $\mathcal{R}_{a}^{i}(\mathcal{A})$.

We now briefly review the basic notions concerning Ershov hiearchy. There is several equivalent definitions of the Ershov hierarchy, introduced in $[4,5,6]$. But our presentation is based on [17].

Definition 1.3. If $a$ is a notation for a computable ordinal, then a set of numbers $A$ is said to be $\Sigma_{a}^{-1}$ if there are a computable function $f(z, t)$ and a partial computable function $\gamma(z, t)$ such that, for all $z$,

1. $A(z)=\lim _{t} f(z, t)$, with $f(z, 0)=0$; (here, given a set $X$, and a number $z$, the symbol $X(z)$ denotes the value of the characteristic function of $X$ on $z)$;

2. (a) $\gamma(z, t) \downarrow \Rightarrow \gamma(z, t+1) \downarrow \& \gamma(z, t+1) \leq_{O} \gamma(z, t)<_{O} a$;

(b) $f(z, t+1) \neq f(z, t) \Rightarrow \gamma(z, t+1) \downarrow \neq \gamma(z, t)$.

We call the partial function $\gamma$ the mind-change function for $A$, relatively to $f$.

A $\Sigma_{a}^{-1}$-approximation to a $\Sigma_{a}^{-1}$-set $A$, is a pair $\langle f, \gamma\rangle$, where $f$ and $\gamma$ are respectively a computable function and a partial computable function satisfying 1. and 2., above, for $A$. 
Following [13], we give the following:

Definition 1.4. A $\Sigma_{a}^{-1}$-computable numbering of a family $\mathcal{A}$ of $\Sigma_{a}^{-1}$-sets is an onto function $\pi: \omega \longrightarrow \mathcal{A}$, such that

$$
\{\langle y, x\rangle: x \in \pi(y)\} \in \Sigma_{a}^{-1} .
$$

Hence there exist a computable function $f(z, t)$ and a partial computable function $\gamma(z, t)$, such that $\pi(y)(x)=\lim _{t} f(\langle y, x\rangle, t)$, with $f(z, 0)=0$ for all $z$; and $\gamma$ is the mind-change function for $\{\langle y, x\rangle: x \in \pi(y)\}$ relatively to $f$.

Note that $\{\langle x, m\rangle: x \in \alpha(m)\} \in \Sigma_{a}^{-1}$ if and only if $\{\langle x, m\rangle: x \in \alpha(m)\}$ is $a$ - computably enumerable in a sense of Putnam [16].

We recall (see e.g. [5]) that there is an indexing $\left\{A_{z}\right\}_{z \in \omega}$ of the family of all $\Sigma_{a}^{-1}$ sets, such that $\left\{\langle x, z\rangle: x \in A_{z}\right\} \in \Sigma_{a}^{-1}$. From this, it is possible (for more details, see [17]) to define an indexing $\left\{\pi_{k}\right\}_{k \in \omega}$ of all computable numberings of families of $\Sigma_{a}^{-1}$ sets, for which

$$
\left\{\langle k, y, x\rangle: x \in \pi_{k}(y)\right\} \in \Sigma_{a}^{-1},
$$

i.e. the set $\left\{\langle k, y, x\rangle: x \in \pi_{k}(y)\right\}$ has a $\Sigma_{a}^{-1}$-approximation $\langle f, \gamma\rangle$ : an indexing satisfying this property is called a $\Sigma_{a}^{-1}$-computable indexing of all $\Sigma_{a}^{-1}$ computable numberings. Clearly, from $k, y$ one has an effective way of getting a $\Sigma_{a}^{-1}$-approximation $\left\langle f_{\pi_{k}(y)}, \gamma_{\pi_{k}(y)}\right\rangle$ to the set $\pi_{k}(y)$.

\section{The theorem}

In [11], S.S. Goncharov showed that there exist classes of recursively enumerable sets admitting up to equivalence exactly one Friedberg numbering which does not induce the least element in the corresponding Rogers semilattice. Later, a simple example of a such a class was found by M. Kummer: This example appears in the paper of S.A. Badaev and S.S. Goncharov ([1]). We generalize this result to all successor ordinal levels of the Ershov hierarchy.

Theorem 2.1. for every ordinal notation a of a nonzero successor ordinal, there exists a $\Sigma_{a}^{-1}$-computable family $\mathcal{A}$ whose Rogers semilattice has exactly one Friedberg degree which is not the least element of the semilattice.

Proof. Given $a$, with $|a|_{O} \geq 1$ and $|a|_{O}$ successor. We will construct a $\Sigma_{a}^{-1}$ computable Friedberg numbering $\alpha$ and a $\Sigma_{a}^{-1}$-computable numbering $\beta$ such that:

1. $\alpha(\omega)=\beta(\omega)$.

2. If $\pi$ is a computable Friedberg numbering of $\mathcal{A}=\alpha(\omega)$ then $\pi \leqslant \alpha$.

3. $\alpha \nless \beta$. 
Requirements. We will define numberings $\alpha$ and $\beta$ so that, for every $e, k$, the following requirements are satisfied:

$\mathcal{F}: \quad \alpha$ is a Friedberg numbering,

$\mathcal{B}: \quad \alpha(\omega)=\beta(\omega)=\mathcal{A}$,

$\mathcal{C}: \quad \alpha$ and $\beta$ are $\Sigma_{a}^{-1}$-computable,

$\mathcal{P}_{k}:$ if $\pi_{k}$ is a Friedberg numbering of $\mathcal{A}$ then $\pi_{k}=\alpha \circ g_{k}$,

$\mathcal{D}_{e}:$ if $\varphi_{e}$ is total then $\alpha \neq \beta \circ \varphi_{e}$,

where $g_{k}$ is a computable function built by us.

Strategy for $\mathcal{F}$. We fix three one-to-one computable functions

$$
a(k, m), b(k, m), c(k, m),
$$

with pairwise disjoint ranges and construct the numbering $\alpha$ so that for every $m$ there exist at least one $k_{m}$ with the following properties, where for simplicity we let $a=a\left(k_{m}, m\right), b=b\left(k_{m}, m\right), c=c\left(k_{m}, m\right)$ :

$$
\begin{aligned}
\alpha(2 m) \cap\{a, b, c\} & =\{a, b, c\}, \\
\alpha(2 m+1) \cap\{a, b, c\} & =\{b, c\}
\end{aligned}
$$

and, for every $m^{\prime} \neq m$,

$$
\alpha\left(2 m^{\prime}\right) \cap\{a, b, c\}=\alpha\left(2 m^{\prime}+1\right) \cap\{a, b, c\}=\emptyset .
$$

Strategy for $\mathcal{B}$. For every $m$, we force $\beta$ to satisfy the following equalities: either, for all $s$,

$$
\begin{aligned}
\beta^{s}(3 m) & =\alpha^{s}(2 m) \\
\beta^{s}(3 m+1) & =\beta^{s}(3 m+2)=\alpha^{s}(2 m+1) ;
\end{aligned}
$$

or there exists $s_{0}$ such that for all $s \leq s_{0}$

$$
\begin{aligned}
\beta^{s}(3 m) & =\alpha^{s}(2 m) \\
\beta^{s}(3 m+1) & =\beta^{s}(3 m+2)=\alpha^{s}(2 m+1)
\end{aligned}
$$

and for all $s>s_{0}$

$$
\begin{aligned}
\beta^{s}(3 m) & =\alpha^{s}(2 m) \\
\beta^{s}(3 m+1) & =\alpha^{s}\left(m^{\prime}\right) \\
\beta^{s}(3 m+2) & =\alpha^{s}\left(m^{\prime \prime}\right) .
\end{aligned}
$$

Here $\left\{m^{\prime}, m^{\prime \prime}\right\}=\{2 m, 2 m+1\}$. 
Strategy for $\mathcal{C}$. To ensure $\Sigma_{a}^{-1}$-computability of the numberings $\alpha$ and $\beta$ we do not explicitly construct suitable corresponding changing functions, but in all the strategies and the construction we implicitly ensure the correct behavior of the approximations to $\alpha$ and $\beta$.

Strategy for $\mathcal{P}_{k}$ in isolation. This strategy aims, for every $m$, at finding by some uniform procedure, two $\pi_{k}$-indices $x \neq y$ such that $\alpha(2 m)=\pi_{k}(x)$ and $\alpha(2 m+1)=\pi_{k}(y)$. In the case when $\pi_{k}$ is a Friedberg numbering of $\mathcal{A}$, this will give us a reduction of $\pi_{k}$ to $\alpha$.

Initially, for all $k \in \omega$, we put $a(k, m), b(k, m), c(k, m)$ into $\alpha(2 m)$, and we put $b(k, m), c(k, m)$ into $\alpha(2 m+1)$. Note that, for every $m, k$, we never remove the numbers $b(k, m), c(k, m)$ from the sets $\alpha(2 m)$ and $\alpha(2 m+1)$.

Due to injectiveness of the functions $a(k, m), b(k, m), c(k, m)$, we can split the strategy for $\mathcal{P}_{k}$ into independent substrategies $\mathcal{P}_{k, m}$, with $m \in \omega$. Henceforth, we write "Substrategy $\mathcal{P}_{k, m}$ " to denote the substrategy for $\mathcal{P}_{k, m}$.

[Note that at any stage of the construction below we use a uniform approximation to the numbering $\pi_{k}$ in which at most one change might happen in $\pi_{k}(x)$ at any stage.]

\section{Substrategy for $\mathcal{P}_{k, m}$ in isolation.}

1. Search for a $\pi_{k}$-index $x$ such that

$$
\pi_{k}(x) \cap\{a(k, m), b(k, m), c(k, m)\}=\{a(k, m), b(k, m), c(k, m)\} .
$$

From now on let $a, b, c$ stand for the numbers $a(k, m), b(k, m), c(k, m)$, respectively.

Furthermore, we check whether $b$ is in $\pi_{k}(x)$ every time we start Substrategy $\mathcal{P}_{k, m}$.

If $b$ is in $\pi_{k}(x)$ then go to item 3 , otherwise go to item 2. In the latter case wait until $b$ comes back to $\pi_{k}(x)$ and only after that continue Substrategy $\mathcal{P}_{k, m}$ in item 3 .

2. So, what should we do when $b \notin \pi_{k}(x)$ ?

Enumerate $b$ into $\alpha(z)$ for all $z \notin\{2 m, 2 m+1\}$ and wait until $b$ appears in $\pi_{k}(x)$, and only when this happens, remove $b$ from $\alpha(z)$ for all $z \notin$ $\{2 m, 2 m+1\}$.

These movements of $b$ from, and into, $\pi_{k}(x)$ eventually stop, and the corresponding synchronized changes of $b$ for $\alpha(z)$ are compatible with having $\alpha(z) \Sigma_{a}^{-1}$-computable. When $\pi_{k}$ is a numbering of $\mathcal{A}$, we have that $b \in \pi_{k}(x) \cap \alpha(2 m) \cap \alpha(2 m+1)$ and $b \notin \alpha(z)$ for all $z \notin\{2 m, 2 m+1\}$. Thus $\pi_{k}(x) \in\{\alpha(2 m), \alpha(2 m+1)\}$. (Note that if $\pi_{k}$ is not a numbering of $\mathcal{A}$ then the option with $b \in \alpha(z)$ for all $z \in \omega$ and $b \notin \pi_{k}(x)$ is possible too.) 
3. $\left[b \in \pi_{k}(x)\right.$ and either $a \in \alpha(2 m)$ or $a \in \alpha(2 m+1)$ : The construction guarantees that at each stage, at the beginning of the current item, $a \in$ $\alpha(2 m)$ if and only if $a \notin \alpha(2 m+1)]$ Then $\pi_{k}(x) \cap\{a\}$ is equal to either $\alpha(2 m) \cap\{a\}$ or $\alpha(2 m+1) \cap\{a\}$. Go to item 4 if $\pi_{k}(x) \cap\{a\}=\alpha(2 m) \cap\{a\}$, and go to item 5 otherwise.

4. $\left[\pi_{k}(x) \cap\{a\}=\alpha(2 m) \cap\{a\}\right]$ Check whether $a$ has exhausted all possible changes in $\pi_{k}(x)$ (i.e. $a \in \pi_{k}(x)$ and $a$ can not be extracted from $\pi_{k}(x)$ anymore, or $a \notin \pi_{k}(x)$ and $a$ can not be put into $\pi_{k}(x)$ anymore). If so then define $g_{k}(x)=2 m$ and go to item 6 , otherwise go to $4 \mathrm{a}$ or to $4 \mathrm{~b}$ according to whether the question "Is $a \in \pi_{k}(x)$ ?" has positive or negative answer.

(a) $\left[a \in \pi_{k}(x)\right]$ Extract $a$ from $\alpha(2 m)$ and wait until $a$ leaves $\pi_{k}(x)$ (if $a$ never leaves $\pi_{k}(x)$ then $\pi_{k}$ is not a numbering of $\mathcal{A}$ since $a$ currently does not belong to any set of $\mathcal{A}$ and we prevent $a$ from being put into any $\alpha(z)$ in the future).

When $a$ leaves $\pi_{k}(x)$, we put $a$ into $\alpha(2 m+1)$ and go to 3 .

(b) $\left[a \notin \pi_{k}(x)\right]$ Put $a$ into $\alpha(2 m)$ (notice that already $a \in \alpha(2 m+1)$ ), and wait until $a$ is enumerated in $\pi_{k}(x)$ (if $a$ never appears in $\pi_{k}(x)$ then $\pi_{k}$ is not a numbering of $\mathcal{A}$.

When $a$ is enumerated in $\pi_{k}(x)$, we remove $a$ from $\alpha(2 m+1)$ and from $\alpha(z)$ for all $z \neq 2 m$, and go to (3).

5. $\left[\pi_{k}(x) \cap\{a\}=\alpha(2 m+1) \cap\{a\}\right]$ Check whether $a$ has exhausted all possible changes in $\pi_{k}(x)$. If so then define $g_{k}(x)=2 m+1$ and go to 6 , otherwise go to $5 \mathrm{a}$ or to $5 \mathrm{~b}$ according to whether the question "Is $a \in \pi_{k}(x)$ ?" has positive or negative answer.

(a) $\left[a \in \pi_{k}(x)\right]$ Extract $a$ from $\alpha(2 m+1)$ and wait until $a$ leaves $\pi_{k}(x)$. When $a$ leaves $\pi_{k}(x)$, we put $a$ into $\alpha(2 m)$, and go to 3 .

(b) $\left[a \notin \pi_{k}(x)\right]$ Put $a$ into $\alpha(2 m+1)$ (notice that already $a \in \alpha(2 m)$ ), and wait until $a$ is enumerated in $\pi_{k}(x)$.

When $a$ is enumerated in $\pi_{k}(x)$, we remove $a$ from $\alpha(2 m)$ and from $\alpha(z)$ for all $z \neq 2 m+1$, and go to 3 .

6. Let $\tilde{m} \in\{2 m, 2 m+1\}$ be such that $g_{k}(x) \neq \tilde{m}$. Our goal now is to find a $\pi_{k}$-index $y \neq x$ such that $\pi_{k}(y)=\alpha(\tilde{m})$ if $\pi_{k}$ is a Friedberg numbering of $\mathcal{A}$.

Search for a $\pi_{k}$-index $y$ such that $y \neq x$ and

$$
\pi_{k}(y) \cap\{a(k, m), b(k, m), c(k, m)\}=\alpha(\tilde{m}) \cap\{a(k, m), b(k, m), c(k, m)\} .
$$

Define $g_{k}(y)=\tilde{m}$.

After this, every time $c(k, m)$ leaves $\pi_{k}(y)$, put $c(k, m)$ into $\alpha(z)$ for all $z \notin\{2 m, 2 m+1\}$, and wait until $c(k, m)$ is enumerated in $\pi_{k}(y)$. Whenever 
the number $c(k, m)$ is enumerated in $\pi_{k}(y)$ remove it from $\alpha(z)$ for all $z \notin\{2 m, 2 m+1\}$.

As a result of item $6, \pi_{k}(y) \in\{\alpha(2 m), \alpha(2 m+1)\}$ if $\pi_{k}$ is a numbering of $\mathcal{A}$ since in this case, for every $z \notin\{2 m, 2 m+1\}$,

$$
c(k, m) \in \pi_{k}(y) \Leftrightarrow c(k, m) \notin \alpha(z) .
$$

Moreover, if $\pi_{k}$ is a Friedberg numbering of $\mathcal{A}$ then $\pi_{k}(y)=\alpha\left(g_{k}(y)\right)$ because $\pi_{k}(x)=\alpha\left(g_{k}(x)\right), x \neq y, g_{k}(x) \neq g_{k}(y)$ and $\left\{\pi_{k}(x), \pi_{k}(y)\right\}=$ $\{\alpha(2 m), \alpha(2 m+1)\}$.

Observations on Substrategy $\mathcal{P}_{k, m}$. It is easy to see that whenever $x$ has been determined, Substrategy $\mathcal{P}_{k, m}$ proceeds through several cycles with starting point at item 3 and possible interruptions of these cycles at item 2. Let $(u, v, w)$ stand for the triple of current total number of changes (enumerations and extractions) of $a=a(k, m)$ relative to the sets $\alpha(2 m), \alpha(2 m+1)$, and $\pi_{k}(x)$ respectively. A closer look at the evolution in time of this triple shows

Lemma 2.2. The following hold

(i) if $\pi_{k}$ is a numbering of $\mathcal{A}$ then eventually $w=n$;

(ii) at the end of every cycle 3 (even when the cycle is not completed because we define $\left.g_{k}\right), u \leq w$ and $v<w$.

Proof. Statement (i) follows from the instructions of items 4a-5b. In each of these four cases, $\pi_{k}(x)$ is forced to move $a$, otherwise $\pi_{k}$ can not be a numbering of $\mathcal{A}$.

In proving (ii), we can ignore item 2 at all, since instructions of item 2 do not change $u$ and $v$ and can not force $w$ to decrease. By analyzing items 4a-5b, it is easy to see that, if at the beginning of any cycle at 3 we have a triple $(u, v, w)$ then a complete cycle, before returning to 3 , may be described by one the following two series of actions, the former due to 4 , and the latter due to 5 :

$$
\begin{aligned}
& (u, v, w) \longrightarrow(u+1, v, w) \longrightarrow(u+1, v, w+1) \longrightarrow(u+1, v+1, w+1), \\
& (u, v, w) \longrightarrow(u, v+1, w) \longrightarrow(u, v+1, w+1) \longrightarrow(u+1, v+1, w+1) .
\end{aligned}
$$

So, if at the beginning of the cycle, $(u, v, w)$ satisfies (ii), then so does at the end of the cycle. The claim then follows from the fact that initially, when we start 3 for the first time, we have $u=1, v=0, w \geq 1$. Notice that 3 may stop before completion of the cycle, if $w=n$ at the beginning of the cycle.

Strategy for $\mathcal{D}_{e}$ in isolation. If $\varphi_{e}$ is total then we diagonalize against the reduction $\alpha=\beta \circ \varphi_{e}$ at the argument $x=2 e+1$. If $\varphi_{e}(2 e+1) \in\{3 e+1,3 e+2\}$ then we define $\beta\left(\varphi_{e}(2 e+1)\right)=\alpha(2 e)$ from the moment when the computation $\varphi_{e}(2 e+1)$ converges. So, up to the stage when $\varphi_{e}(2 e+1)$ becomes defined, both $\beta(3 e+1)$ and $\beta(3 e+2)$ behave like $\alpha(2 e+1)$, but after that stage the 
set $\beta\left(\varphi_{e}(2 e+1)\right)$ behaves as $\alpha(2 e)$ while the second one continues to behave as $\alpha(2 e+1)$.

The main idea here is to exploit the possibility to transform

$$
\alpha(2 e+1) \cap\{a(k, e), b(k, e), c(k, e)\}
$$

into

$$
\alpha(2 e) \cap\{a(k, e), b(k, e), c(k, e)\},
$$

for all $k$, uniformly. This means that, at the moment when $\beta\left(\varphi_{e}(2 e+1)\right)$ switches from behaving like $\alpha(2 e+1)$ to behave like $\alpha(2 e)$, every $x \in \alpha(2 e+1)$ must have at its disposal the possibility of changing its membership status from $\alpha(2 e+1)(x)$ to $\alpha(2 e)(x)$. In isolation, $\mathcal{D}_{e}$ can easily achieve this: To this end, notice also, that the total number of membership changes of every $x \neq a(k, e)$, relative to $\alpha(2 e+1)$ and $\alpha(2 e)$, are the same. So, we only need to control the changes of $a(k, e)$, with $k \in \omega$.

In details, the strategy for $\mathcal{D}_{e}$ (henceforth referred to also as "Strategy $\mathcal{D}_{e}$ ") in isolation proceeds as follows.

1. Wait for the computation $\varphi_{e}(2 e+1)$ to be defined. If $\varphi_{e}(2 e+1) \notin\{3 e+$ $1,3 e+2\}$ then do nothing, since in this case, evidently, $\alpha(2 e+1) \neq$ $\beta\left(\varphi_{e}(2 e+1)\right)$. Otherwise,

2. Wait until, for every $k$, if Substrategy $\mathcal{P}_{k, e}$ has acted relatively to $a(k, e)$ then at least one of $2 e$ or $2 e+1$ has been already put into the range of $g_{k}$.

3. If we successfully stop waiting for every $k$, make $\beta\left(\varphi_{e}(2 e+1)\right)$ equal to the current $\alpha(2 e)$ as follows:

(a) if $a(k, e) \in \alpha(2 e) \backslash \alpha(2 e+1)$ then enumerate $a(k, e)$ into $\beta\left(\varphi_{e}(2 e+1)\right)$;

(b) if $a(k, e) \in \alpha(2 e+1) \backslash \alpha(2 e)$ then remove $a(k, e)$ from $\beta\left(\varphi_{e}(2 e+1)\right)$.

4. After 3 is done, do not touch anymore $a(k, e), k \in \omega$, in any of the sets $\alpha(2 e), \alpha(2 e+1), \beta(3 e), \beta(3 e+1), \beta(3 e+2)$.

Interactions between strategies. Obviously, there is no interference between the various substrategies $\mathcal{P}_{k, m}$, for $k, m \in \omega$. Strategies $\mathcal{D}_{e}$, with $e \in \omega$, are pairwise independent too since, if $e \neq e^{\prime}$, then we diagonalize against reductions of $\alpha$ to $\beta$ via $\varphi_{e}$ and $\varphi_{e^{\prime}}$, respectively, on different $\alpha$-indices.

No substrategy $\mathcal{P}_{k, m}, k \in \omega$, conflicts with Strategy $\mathcal{D}_{e}$, if $m \neq e$, since they deal with disjoint pairs of sets, namely, with the pair $\alpha(2 m), \alpha(2 m+1)$, and the pair $\alpha(2 e), \alpha(2 e+1)$, respectively.

Strategy $\mathcal{D}_{e}$ can conflict with Substrategy $\mathcal{P}_{k, e}$ for an isolated $k$, or meet an infinite series of conflicts with the sequence consisting of Substrategies $\mathcal{P}_{k, e}$, $k \in \omega$. The sequence $\mathcal{P}_{k, e}, k \in \omega$, might prevent Strategy $\mathcal{D}_{e}$ from succeeding, because it might cause $\mathcal{D}_{e}$ to wait forever in item 3 because of the following reasons: 
- some substrategy $\mathcal{P}_{k^{\prime}, e}$ has acted, using $a\left(k^{\prime}, e\right)$, before the moment when $\varphi_{e}(2 e+1)$ has converged, but $\mathcal{P}_{k^{\prime}, e}$ does not achieve its goal, i.e. neither of $2 e, 2 e+1$ becomes a value of $g_{k^{\prime}}$ (so, $\mathcal{P}_{k^{\prime}, e}$ might want to move again $a\left(k^{\prime}, e\right)$, conflicting with 4 of $\left.\mathcal{D}_{e}\right)$; or

- each $\mathcal{P}_{k, e}, k \in \omega$, achieves its goal, but at any stage after convergence of the computation $\varphi_{e}(2 e+1)$ there is at least one $\mathcal{P}_{k, e}$ which has already acted with $a(k, e)$, but is still in progress, i.e. has not as yet contributed to the definition of $g_{k}$.

To resolve these conflicts we use, in Substrategy $\mathcal{P}_{k, m}$, two triples of functions $a_{i}(k, m), b_{i}(k, m), c_{i}(k, m)$, with $i \in\{0,1\}$ instead of just a single triple of functions $a(k, m), b(k, m), c(k, m)$ as above. The subscript $i$ is considered as a switch for using one triple or another.

For a given $k, m$, we start with option $i=0$. This means that we implement the instructions of Substrategy $\mathcal{P}_{k, m}$, with the functions $a(k, m), b(k, m), c(k, m)$ replaced by the functions $a_{0}(k, m), b_{0}(k, m), c_{0}(k, m)$, respectively. We carry out this option until $\varphi_{m}(2 m+1)$ is defined (if ever) at a stage $s_{m}$. We continue option $i=0$ forever if $\varphi_{m}(2 m+1) \notin\{2 m, 2 m+1\}$. Otherwise, we go to the end of stage $s_{m}$ and transform $\beta\left(\varphi_{m}(2 m+1)\right)$ into $\alpha(2 m)$ as in item 3 of Strategy $\mathcal{D}_{m}$; but for all $k \in \omega$, starting from stage $s_{m}+1$ we switch to option $i=1$, i.e. we carry out the instructions of Substrategy $\mathcal{P}_{k, m}$ operating with $a_{1}(k, m)$, $b_{1}(k, m), c_{1}(k, m)$ from the very beginning but only for those $k$ such that by stage $s_{m}$ the range of the function $g_{k}$ is disjoint from $\{2 m, 2 m+1\}$ : In this case, from stage $s_{m}+1$ the functions $a_{0}(k, m), b_{0}(k, m), c_{0}(k, m)$ play a passive role while proceeding under option $i=1$.

We assume that all these six functions above are injective and have pairwise disjoint ranges.

Given $m$, we build approximations to the sets $\alpha(2 m), \alpha(2 m+1)$ uniformly in $m$ by a stage construction. Approximations to the sets $\beta(3 m), \beta(3 m+1)$, $\beta(3 m+2)$ are built essentially from the approximations for $\alpha(2 m), \alpha(2 m+1)$. We define simultaneously the sequence $g_{k}$, with $k \in \omega$, of partial computable functions, or, to be more precise, the preimages of $g_{k}$ on the set $\{2 m, 2 m+1\}$. We denote the option used for constructing the sets $\alpha(2 m), \alpha(2 m+1)$ at stage $s$, by $i^{s}(m)$.

The construction. The construction is by stages. At each stage, if not explicitly redefined, every parameter is understood to retain the same value as at the previous stage.

Stage 0. Let $i^{0}(m)=0$,

$$
\begin{gathered}
f_{\alpha}(2 m, x, 0)=1 \text { with } \gamma_{\alpha}(2 m, x, 0)=a ; \quad f_{\alpha}\left(2 m+1, a_{0}(k, m), 0\right)=0 \text { with } \gamma_{\alpha}\left(2 m+1, a_{0}(k, m), 0\right) \uparrow ; \\
f_{\alpha}(2 m+1, \varrho, 0)=1 \text { with } \gamma_{\alpha}(2 m+1, \varrho, 0)=a \\
\text { where } x \in\left\{a_{0}(k, m), b_{0}(k, m), c_{0}(k, m)\right\} \text { and } \varrho \in\left\{b_{0}(k, m), c_{0}(k, m)\right\}
\end{gathered}
$$


So, we put $a_{0}, b_{0}, c_{0}$ to $\alpha(2 m)$ and put $b_{0}, c_{0}$ to $\alpha(2 m+1)$ with maximal change $a$. Go to stage 1 .

Stage $s+1$. Let $s=\langle k, m, t\rangle$. At the beginning of stage $s+1$ we decide on the option $i=i^{s+1}(m)$, and execute the instructions of Substrategy $\mathcal{P}_{k, m}^{i}$, i.e. Substrategy $\mathcal{P}_{k, m}$, but relative to option $i$. We split the instructions of Substrategy $\mathcal{P}_{k, m}$ into mutually exclusive parts, named Procedure $\mathcal{I}_{k, m}^{i}$ and Procedure $\mathcal{P}_{k, m}^{i, j}$, where $i$ is the current option, and $j \leq 2$ is a parameter relative to the procedure, connected with the cardinality of $\operatorname{range}\left(g_{k}^{s}\right) \cap\{2 m, 2 m+1\}$.

Procedure $\mathcal{I}_{k, m}^{i}$ corresponds to the instructions of item 2 of Substrategy $\mathcal{P}_{k, m}$, while Procedures $\mathcal{P}_{k, m}^{i, j}$, with $j \leq 2$, correspond to items 3-6 of Substrategy $\mathcal{P}_{k, m}$. We complete stage $s+1$ with the procedure End of Stage which aims at constructing the sets $\beta(3 m), \beta(3 m+1), \beta(3 m+2)$. Thus Strategy $\mathcal{D}_{m}$ is in fact implemented in End of Stage.

In order to decide on the option $i$, we go through stage $s+1$ by checking whether $\varphi_{m}^{s+1}(2 m+1)$ is defined, and $\varphi_{m}(2 m+1)$ is in $\{3 m+1,3 m+2\}$. If this is not the case then we let $i^{s+1}(m)=0$ and go to Procedure $\mathcal{I}_{k, m}^{0}$, otherwise we let $i^{s+1}(m)=1$. If $i^{s}(m)=0$ then we go to End of Stage, otherwise we go to Procedure $\mathcal{I}_{k, m}^{1}$.

For the sake of simplicity, let $a_{i}=a_{i}(k, m), b_{i}=b_{i}(k, m)$, and $c_{i}=c_{i}(k, m)$; we denote by $x_{i}, y_{i}$ the $\pi_{k}$-indices possibly determined by Procedures $\mathcal{P}_{k, m}^{i, j}$.

Procedure $\mathcal{I}_{k, m}^{i}$. If $x_{i}$ and $b_{i}$ have not been determined by Procedure $\mathcal{P}_{k, m}^{i, 0}$ then go to Procedure $\mathcal{P}_{k, m}^{i, 0}$, otherwise execute the instructions of one of the following cases.

1. If $f_{\pi}\left(k, x_{i}, b_{i}, s+1\right)=1$ with $\gamma_{\pi}\left(k, b_{i}, x_{i}, s+1\right) \downarrow$ and $f_{\alpha}\left(2 m+2, b_{i}, s+1\right)=0$ then go to Procedure $\mathcal{P}_{k, m}^{i, j}$, for the relevant $j$.

2. If $f_{\pi}\left(k, x_{i}, b_{i}, s+1\right)=0$ and $f_{\alpha}\left(2 m+2, b_{i}, s+1\right)=0$ then enumerate $b_{i}$ into $\alpha(z)$ for all $z \notin\{2 m, 2 m+1\}$ with $\gamma_{\alpha}\left(z, b_{i}, s+1\right)=\gamma_{\pi}\left(k, x_{i}, b_{i}, s+1\right)$ and go to the next stage.

3. If $f_{\pi}\left(k, x_{i}, b_{i}, s+1\right)=0$ and $f_{\alpha}\left(2 m+2, b_{i}, s+1\right)=1$ with $\gamma_{\alpha}\left(2 m+2, b_{i}, s+\right.$ $1)=\gamma_{\pi}\left(k, x_{i}, b_{i}, s+1\right)$, then go to the next stage.

4. If $f_{\pi}\left(k, x_{i}, b_{i}, s+1\right)=1$ and $f_{\alpha}\left(2 m+2, b_{i}, s+1\right)=1$ then extract $b_{i}$ from $\alpha(z)$ for all $z \notin\{2 m, 2 m+1\}$ and go to Procedure $\mathcal{P}_{k, m}^{i, j}$, for the relevant $j$.

Procedure $\mathcal{P}_{k, m}^{i, 0}$. This procedure is executed if $a_{i}, b_{i}, c_{i}$ have been chosen but a $\pi_{k}$-index $x_{i}$ has not been determined.

Search for $x$ such that $f_{\pi}\left(k, x_{i}, q_{i}, s+1\right)=1$ for all $q_{i}$, where $q_{i} \in\left\{a_{i}, b_{i}, c_{i}\right\}$.

If $x$ exists then denote by $x_{i}$ the least such $x$, go to Procedure $\mathcal{P}_{k, m}^{i, 1}$; otherwise go to the next stage. 
Procedure $\mathcal{P}_{k, m}^{i, 1}$. This procedure is executed if neither $2 m$ nor $2 m+1$ is in the range of $g_{k}$ and if $a_{i}$ and a $\pi_{k}$-index $x_{i}$ have been chosen in Procedure $\mathcal{P}_{k, m}^{i, 0}$.

If $a_{i}$ does exhaust all possible changes in $\pi_{k}\left(x_{i}\right)$ then define

$$
g_{k}\left(x_{i}\right)= \begin{cases}2 m, & \text { if } f_{\pi}\left(k, x_{i}, a_{i}, s+1\right)=f_{\alpha}\left(2 m, a_{i}, s\right), \\ 2 m+1, & \text { if } f_{\pi}\left(k, x_{i}, a_{i}, s+1\right)=f_{\alpha}\left(2 m+1, a_{i}, s\right),\end{cases}
$$

and go to Procedure $\mathcal{P}_{k, m}^{i, 2}$.

If $a_{i}$ does not exhaust all possible changes in $\pi_{k}\left(x_{i}\right)$ then execute one of the following nine mutually exclusive cases and after that go to the next stage.

1. If $f_{\alpha}\left(2 m, a_{i}, s\right)=f_{\pi}\left(k, x_{i}, a_{i}, s+1\right)=1$ and $f_{\alpha}\left(2 m+1, a_{i}, s\right)=0$ then extract $a_{i}$ from $\alpha(2 m)$.

2. If $f_{\alpha}\left(2 m, a_{i}, s\right)=f_{\alpha}\left(2 m+1, a_{i}, s\right)=f_{\pi}\left(k, x_{i}, a_{i}, s+1\right)=0$ then enumerate $a_{i}$ into $\alpha(2 m+1)$.

3. If $f_{\alpha}\left(2 m, a_{i}, s\right)=f_{\pi}\left(k, x_{i}, a_{i}, s+1\right)=0$ and $f_{\alpha}\left(2 m+1, a_{i}, s\right)=1$ then enumerate $a_{i}$ into $\alpha(2 m)$.

4. If $f_{\alpha}\left(2 m, a_{i}, s\right)=f_{\pi}\left(k, x_{i}, a_{i}, s+1\right)=f_{\alpha}\left(2 m+1, a_{i}, s\right)=f_{\alpha}(2 m+$ $\left.2, a_{i}, s\right)=1$ then remove $a_{i}$ from $\alpha(2 m+1)$.

5. If $f_{\pi}\left(k, x_{i}, a_{i}, s+1\right)=f_{\alpha}\left(2 m+1, a_{i}, s\right)=1$ and $f_{\alpha}\left(2 m, a_{i}, s\right)=0$ then extract $a_{i}$ from $\alpha(2 m+1)$.

6. If $f_{\alpha}\left(2 m, a_{i}, s\right)=f_{\pi}\left(k, x_{i}, a_{i}, s+1\right)=f_{\alpha}\left(2 m+1, a_{i}, s\right)=0$ then enumerate $a_{i}$ into $\alpha(2 m)$.

7. If $f_{\alpha}\left(2 m+1, a_{i}, s\right)=f_{\pi}\left(k, x_{i}, a_{i}, s+1\right)=0$ and $f_{\alpha}\left(2 m, a_{i}, s\right)=1$ then enumerate $a_{i}$ into $\alpha(2 m+1)$.

8. If $f_{\alpha}\left(2 m, a_{i}, s\right)=f_{\pi}\left(k, x_{i}, a_{i}, s+1\right)=f_{\alpha}\left(2 m+1, a_{i}, s\right)=f_{\alpha}(2 m+$ $\left.2, a_{i}, s\right)=1$ then remove $a_{i}$ from $\alpha(2 m)$.

9. Cases 1-8 do not hold. Do nothing, just go to the next stage.

Procedure $\mathcal{P}_{k, m}^{i, 2}$. This procedure is executed when $a_{i}, b_{i}, c_{i}$ have been chosen and exactly one of numbers $2 m$ or $2 m+1$ is in the range of $g_{k}$. Let $\tilde{m} \in$ $\{2 m, 2 m+1\}$ be the number which still is not in the range of $g_{k}$. The procedure includes $\tilde{m}$ into the range of $g_{k}$, and after that it continues to control correctness of $\tilde{m}$ as a value of $g_{k}$. This procedure corresponds to item 6 of Substrategy $\mathcal{P}_{k, m}$, and consists of the following 4 mutually exclusive cases.

1. $\tilde{m} \notin \operatorname{range}\left(g_{k}^{s}\right)$ and there exists $y$ such that $y \neq x$ and $f_{\pi}\left(k, y, q_{i}, s+1\right)=$ $f_{\alpha}\left(\widetilde{m}, q_{i}, s\right)$ for all $q_{i}$, where $q_{i} \in\left\{a_{i}, b_{i}, c_{i}\right\}$. Let $y_{i}$ stand for the least such $y$. Define $g_{k}\left(y_{i}\right)=\tilde{m}$ and go the next stage.

2. $g_{k}\left(y_{i}\right)=\tilde{m}$ and $f_{\pi}\left(k, y_{i}, c_{i}, s+1\right)=f_{\alpha}\left(2 m+2, c_{i}, s\right)=0$. Enumerate $c_{i}$ into $\alpha(z)$ for all $z \notin\{2 m, 2 m+1\}$ and go to the next stage. 
3. $g_{k}\left(y_{i}\right)=\tilde{m}$ and $f_{\pi}\left(k, y_{i}, c_{i}, s+1\right)=f_{\alpha}\left(2 m+2, c_{i}, s\right)=1$.

Remove $c_{i}$ from $\alpha(z)$ for all $z \notin\{2 m, 2 m+1\}$ and go to the next stage.

4. Cases 1-3 do not hold. Do nothing, just go to the next stage.

End of Stage. If $\varphi_{m}^{s+1}(2 m+1)$ is defined and $\varphi_{m}(2 m+1) \in\{3 m+1,3 m+2\}$ then denote $\varphi_{m}(2 m+1)=m^{\prime}$, and denote the number from $\{3 m+1,3 m+2\}$ different from $m^{\prime}$ by $m^{\prime \prime}$.

1. If $i^{s+1}(m)=0$ then define

$$
\begin{aligned}
\beta^{s+1}(3 m) & =\alpha^{s+1}(2 m), \\
\beta^{s+1}(3 m+1) & =\beta^{s+1}(3 m+2)=\alpha^{s+1}(2 m+1) .
\end{aligned}
$$

2. If $i^{s+1}(m)=i^{s}(k, m)=1$ then define

$$
\begin{aligned}
& \beta^{s+1}(3 m)=\beta^{s+1}\left(m^{\prime}\right)=\alpha^{s+1}(2 m), \\
& \beta^{s+1}\left(m^{\prime \prime}\right)=\alpha^{s+1}(2 m+1) .
\end{aligned}
$$

3. If $i^{s+1}(m)=1$ but $i^{s}(m)=0$ then define

$$
\begin{aligned}
& \beta^{s+1}(3 m)=\beta^{s+1}\left(m^{\prime}\right)=\alpha^{s+1}(2 m) \\
& \beta^{s+1}\left(m^{\prime \prime}\right)=\alpha^{s+1}(2 m+1),
\end{aligned}
$$

where

$$
\begin{aligned}
& \alpha^{s+1}(2 m)= \\
& \alpha^{s}(2 m) \cup\left\{a_{1}(l, m), b_{1}(l, m), c_{1}(l, m) \mid \operatorname{range}\left(g_{l}^{s}\right) \cap\{2 m, 2 m+1\}=\emptyset\right\}
\end{aligned}
$$

and

$$
\begin{aligned}
& \alpha^{s+1}(2 m+1)= \\
& \quad \alpha^{s}(2 m+1) \cup\left\{b_{1}(l, m), c_{1}(l, m) \mid \operatorname{range}\left(g_{l}^{s}\right) \cap\{2 m, 2 m+1\}=\emptyset\right\} .
\end{aligned}
$$

(Notice that we never choose to operate with either of $a_{1}(l, m), b_{1}(l, m)$, $c_{1}(l, m)$ if range $\left(g_{l}^{s}\right) \cap\{2 m, 2 m+1\} \neq \emptyset$. In other words, once the first definition has been made for $g_{l}$ by Procedure $\mathcal{P}_{l, m}^{0,1}$, the procedure does not need to move again $a_{0}(l, m)$, which then can freely change its membership status in $\beta\left(m^{\prime \prime}\right)$ from that of $\alpha(2 m+1)$ to that of $\left.\alpha(2 m)\right)$.

Go to the next stage. 
Verification. By Lemma 2.2, and Procedure End of Stage, we have that $\alpha$ and $\beta$ are $\Sigma_{a}^{-1}$-computable numberings of the same family $\mathcal{A}=\alpha(\omega)$.

Lemma 2.3. $\alpha \not \leq \beta$.

Proof. Suppose that $\varphi_{m}$ is total, and $\varphi_{m}(2 m+1)=m^{\prime} \in\{3 m+1,3 m+2\}$. Let $s+1$ be the least stage at which $i^{s+1}(m)=1$. Since up to this stage (i.e. at all stages $t \leq s)$ we had $\beta^{t}\left(m^{\prime}\right)=\alpha^{t}(2 m+1)$, by Lemma 2.2 we have that for every $k$ such that range $\left(g_{k}^{s}\right) \cap\{2 m, 2 m+1\} \neq \emptyset$, the number $v$ of changes of $a_{0}(k, m)$ in the approximation to $\beta\left(m^{\prime}\right)$ up to stage $s$, is $v<n$, so we can afford to change $\beta^{s+1}\left(m^{\prime}\right)\left(a_{0}(k, m)\right)$ if needed, in order to switch $\beta\left(m^{\prime \prime}\right)$ to $\alpha(2 m)$.

Since $\alpha \neq \beta \circ \varphi_{m}$, for every total $\varphi$, we have that $\alpha \not \leq \beta$.

Lemma 2.4. For every $m$, there exist $k_{m}$ such that, letting $a=a_{0}\left(k_{m}, m\right), b=$ $b_{0}\left(k_{m}, m\right), c=c_{0}\left(k_{m}, m\right)$, we have that $\{a, b, c\} \subseteq \alpha(2 m), \alpha(2 m+1) \cap\{a, b, c\}=$ $\{b, c\}$, and for every $m^{\prime} \neq m, \alpha\left(2 m^{\prime}\right) \cap\{a, b, c\}=\alpha\left(2 m^{\prime}+1\right) \cap\{a, b, c\}=\emptyset$.

Proof. Without loss of generality, we can assume that $\pi_{0}$ is a numbering of the family $\{\emptyset\}$ and that $\pi_{0}^{s}(x)=\emptyset$ for all $s, x \in \omega$. Then, for every $m$, at stage 0 , numbers $a_{0}(0, m), b_{0}(0, m), c_{0}(0, m)$ are enumerated into $\alpha(2 m+1)$ while the numbers $b_{0}(0, m), c_{0}(0, m)$ are enumerated into $\alpha(2 m)$. Since $\pi_{0}^{s}(x)=\emptyset$ for all $s, x \in \omega$ it follows that we will never deal with Procedures $\mathcal{P}_{k, m}^{0, j}$ for $j \geq 1$. Therefore, we will not operate with numbers $a_{0}(0, m), b_{0}(0, m), c_{0}(0, m)$ at all stages $s>0$, so their mebership status relative to any $\alpha(z)$ never changes.

In particular,

Corollary 2.5. $\alpha$ is a Friedberg numbering.

Proof. Immediate.

Lemma 2.6. For every $k$, if $\pi_{k}$ is a numbering of $\mathcal{A}$ then range $\left(g_{k}\right)=\omega$.

Proof. Assume that $\pi_{k}$ is a numbering of $\mathcal{A}$, and let $m \in \omega$. We show in this case that $\{2 m, 2 m+1\} \subseteq$ range $\left(g_{k}\right)$. We distinguish the following two cases.

Case 1: $i^{s}(m)=0$ for every $s$. Thus we implement Substrategy $\mathcal{P}_{k, m}$, at each stage of the form $\langle k, m, t\rangle$, by executing only procedures relative to option $i=0$, operating on the elements $a_{0}=a_{0}(k, m), b_{0}=b_{0}(k, m), c_{0}=$ $c_{0}(k, m)$. Since $\pi_{k}$ is a numbering of the family, Procedure $\mathcal{P}_{k, m}^{0,0}$ gives eventually a successful number $x_{0}$ : Otherwise, for every $s$, we would have $a_{0}, b_{0}, c_{0} \in$ $\alpha^{s}(2 m)$ but for every $x, s, \pi_{k}^{s}(x) \cap\left\{a_{0}, b_{0}, c_{0}\right\} \neq\left\{a_{0}, b_{0}, c_{0}\right\}$. Similarly, Procedure $\mathcal{I}_{k, m}^{0}$ eventually exits, after its last execution, at item 1 or item 4 , the other outcomes providing $b_{0} \in \alpha(z) \backslash \pi_{k}(x)$, for all $z$ : Notice that item 1 or item 4 give that $b_{0} \in \pi_{k}(x) \cap \alpha(2 m) \cap \alpha(2 m+1)$ and $b_{0} \notin \alpha(z)$ for every $z \notin\{2 m, 2 m+1\}$. After last execution of $\mathcal{I}_{k, m}^{0}$, at stages of the form $\langle k, m, t\rangle$ we execute procedure $\mathcal{P}_{k, m}^{0,1}$. Since $\pi_{k}$ is a numbering of $\mathcal{A}$, by Lemma 2.2 the only possible exits for this procedure are when $\pi_{k}(x)\left(a_{0}\right)$ has made $n$ changes, and we define $g_{k}\left(x_{0}\right)=$ $2 m$, or $g_{k}\left(x_{0}\right)=2 m+1$. After this, again at stages of the form $\langle k, m, t\rangle$, we execute Procedure $\mathcal{P}_{k, m}^{0,2}$ : Since $\pi_{k}$ is a numbering of $\mathcal{A}$, the procedure exits with 
determining a number $y_{0} \neq x_{0}$ such that $g_{k}\left(y_{0}\right)=\tilde{m}$, with $\tilde{m} \in\{2 m, 2 m+1\}$ such that $g_{k}\left(x_{0}\right) \neq \tilde{m}$ : Such a number $y_{0}$ exists since $\alpha(2 m) \neq \alpha(2 m+1)$ by Corollary 2.5.

Case 2: There exists a least stage $s+1$ such that $i^{s+1}(m)=1$. Up to, and including, stage $s$, we have already put some pairs $\left\{2 m^{\prime}, 2 m^{\prime}+1\right\}$ in the range of $g_{k}$; or for some $m^{\prime \prime}$ we have put only one of $\left\{2 m^{\prime \prime}, 2 m^{\prime \prime}+1\right\}$ in the range of $g_{k}$ : If, say, we have put only $2 m^{\prime}$ in the range of $g_{k}$, then arguing as in Case 1, and using the fact that $\pi_{k}$ is a numbering of the family, we conclude that Procedure $\mathcal{P}_{k, m}^{0,2}$ eventually finishes off its job, by putting also $2 m^{\prime \prime}+1$ in the range of $g_{k}$. A similar argument applies if by stage $s$ we have put only $2 m^{\prime \prime}+1$ in the range of $g_{k}$. For all other numbers $m$ (those for which range $\left.\left(g_{k}^{s}\right) \cap\{2 m, 2 m+1\}=\emptyset\right)$, starting from $\mathrm{s}+1$ we stop executing Procedures $\mathcal{P}_{k, m}^{0,0}, \mathcal{I}_{k, m}^{0}, \mathcal{P}_{k, m}^{0,1}, \mathcal{P}_{k, m}^{0,2}$, and we execute instead $\mathcal{P}_{k, m}^{1,0}, \mathcal{I}_{k, m}^{1}, \mathcal{P}_{k, m}^{1,1}, \mathcal{P}_{k, m}^{1,2}$. An argument similar to Case 1 allows us to conclude that, by operating with the elements $a_{1}=a_{1}(k, m), b_{1}=b_{1}(k, m), c_{1}=c_{1}(k, m)$, one eventually defines $\left\{g_{k}\left(x_{1}\right), g_{k}\left(y_{1}\right)\right\}=\{2 m, 2 m+1\}$ for suitable numbers $x_{1}, y_{1}$ determined by Procedures $\mathcal{P}_{k, m}^{1,0}, \mathcal{P}_{k, m}^{1,1}$, and $\mathcal{P}_{k, m}^{1,2}$.

Corollary 2.7. For every $k$, if $\pi_{k}$ is a Friedberg numbering of $\mathcal{A}$ then $g_{k}$ is a total function and $g_{k}$ reduces $\pi_{k}$ to $\alpha$.

Proof. Suppose that $\pi_{k}$ is a Friedberg numbering of $\mathcal{A}$. If after executing for the last time Procedure $\mathcal{P}_{k, m}^{i, 1}$, we define $g_{k}\left(x_{i}\right)=m^{\prime}$, with $m^{\prime} \in\{2 m, 2 m+1\}$, and after executing for the last time Procedure $\mathcal{P}_{k, m}^{i, 2}$, we define $g_{k}\left(y_{i}\right)=m^{\prime \prime}$, with $y_{i} \neq x_{i}, m^{\prime \prime} \neq m^{\prime}$, and $m^{\prime \prime} \in\{2 m, 2 m+1\}$, then we can argue that $\pi_{k}\left(y_{i}\right)=\alpha\left(m^{\prime \prime}\right)$ as follows. Operating on $c_{i}$ (by extracting or enumerating $c_{i}$ into $\alpha(z))$ we make sure that $\pi_{k}\left(y_{i}\right)\left(c_{i}\right) \neq \alpha(z)\left(c_{i}\right)$ for every $z \notin\{2 m, 2 m+1\}$. Thus $\pi_{k}\left(y_{i}\right) \in\{\alpha(2 m), \alpha(2 m+1)\}$ : Since $\pi_{k}$ is Friedberg and $y_{i} \neq x_{i}$, we conclude that $\pi_{k}\left(y_{i}\right)=\alpha\left(m^{\prime \prime}\right)$.

Finally let us show that $g_{k}$ is total: for every $x$, there exists $m$ such that $\pi_{k}(x)=\alpha(m)$, but since $\pi_{k}$ is Friedberg there is no $y \neq x$ such that $\pi_{k}(x)=$ $\alpha(m)$. So when we put $m \in \operatorname{range}\left(g_{k}\right)$ we in fact define $g_{k}(x)=m$.

\section{References}

[1] S.A. Badaev, S.S. Goncharov. On computable minimal enumerations,.In Algebra. Proceedings of the Third Internation-al Conference on Algebra, Dedicated to the Memory of M.I. Kargopolov. Krasnoyarsk, August 23-28, 1993.- Walter de Gruyter, Berlin- New York, 1995, pp 21-32

[2] Badaev, Serikzhan A. and Goncharov, Sergey S., The theory of numberings: open problems, in: "Computability theory and its applications. Current trends and open problems" (eds. Cholak, Peter A.; Lempp, Steffen; Lerman, 
Manuel; and Shore, Richard A.), Amer. Math. Soc., Providence, RI, 2000, $23-38$.

[3] Yu.L. Ershov. Numberings of the families of total recursive functions, Sib. Math. J., 1967, v.8, n.5., pp.1015-1025 (Russian).

[4] Yu.L. Ershov, On a hierarchy of sets, I. Algebra i Logika, 1968, v.7, n.1, pp,47-74 (Russian).

[5] Yu.L. Ershov, On a hierarchy of sets, II. Algebra i Logika, 1968, v.7, n.4, pp,15-47 (Russian).

[6] Yu.L. Ershov, On a hierarchy of sets, III. Algebra i Logika, 1970, v.9, n.1, pp,20-31 (Russian).

[7] Yu.L. Ershov, Theory of numberings.-Nauka, Moscow, 1977 (Russian).

[8] Yu.L. Ershov, Theorie der Numerierungen. Z. Math. Logik Grundlagen Math., 1977, v.23, pp.289-371.

[9] , S.S. Goncharov.Computable single-valued numerations. Algebra and Logic, 1980, v.19, n.5, pp,325-356.

[10] R.M. Friedberg, Three theorems on recursive enumeration. J. Symbolic Logic, 1958, v.23, n.3, pp.309-316.

[11] S.S. Goncharov. The family with unique univalent but not the smallest enumeration. Trudy Inst. Matem. SO AN SSSR, v.8, pp.42-48, Nauka, Novosibirsk, 1988 (Russian).

[12] Goncharov, S. S. Problem of the number of non-self-equivalent constructivizations. Algebra and Logic, v.19, n.6(1980), 401-414.

[13] S.S. Goncharov, A. Sorbi, Generalized computable numerations and nontrivial Rogers semilattices. Algebra and Logic, 1997, v.36, n.4., pp.359-369.

[14] , Yu.L. Ershov,Goncharov, S. S..Constructive models. Transl. from the Russian. (English) (Siberian School of Algebra and Logic) Siberian School of Algebra and Logic. New York, NY: Consultants Bureau. xii, 293 p. (2000) 1980.

[15] , M. Kummer.Some applications of computable one-one numberings. Arch. Math. Log. v.30, n.4 (1990), 219-230.

[16] H. Putnam, Trial and error predicates and the solution to a problem of Mostowski. J. Symbolic Logic, 1965, v.30, no.1, 49-57.

[17] S. Ospichev. Computable family of $\Sigma_{a}^{-1}$-sets without Friedberg numberings. In 6th Conference on Computability in Eueope, CiE 2010, 6th Conference on Computability in Europe, CiE 2010. Ponta Delgada, Azores, pages 311315,2010 . 
[18] H. Rogers, Jr. Theory of Recursive Functions and Effective Computability. McGraw-Hill, New York, 1967. 